\title{
Características sociodemográficas de las cuidadoras y problemas que enfrentan en la atención del niño con asma
}

\author{
Georgina Eugenia Bazán-Riverón, * ${ }^{*}$ Jorge Iván Rodríguez Martínez, ${ }^{*}$ Maricela Osorio Guzmán, * \\ Javier Sandoval-Navarrete ${ }^{\S}$
}

\author{
*Facultad de Estudios Superiores Iztacala, UNAM. México. Miembro del Proyecto de Enfermedades Crónicas; \\ ${ }^{\ddagger}$ Facultad de Estudios Superiores Iztacala, UNAM. México. Colaborador Externo del Proyecto de Enfermedades Crónicas; \\ ${ }^{\S}$ Facultad de Medicina, UNAM. México. \\ Trabajo recibido: 25-VII-2013; aceptado: 09-I-2014
}

\begin{abstract}
RESUMEN. Este estudio evaluó en madres cuidadoras de niños con asma descontrolada, la percepción parental de síntomas, el impacto familiar del asma y aspectos sociodemográficos relacionados con los problemas para seguir las prescripciones médicas. Participaron 110 cuidadoras primarias (edad media $=37.6$ años; DT $=6.7$ ) y sus hijos con asma (edades entre 7 y 15 años; media $=12.3$ años; DT $=2.9$ ). Los resultados mostraron que las familias son de clase social desfavorecida, y que la percepción de síntomas se presenta en porcentaje elevado (95.6\%). Las cuidadoras mostraron un fuerte impacto en su vida a nivel funcional y emocional (91\%). En cuanto a los aspectos sociodemográficos son mujeres con pobre desarrollo profesional que han abandonado su vida laboral formal, los padres participan poco y ellas reciben apoyo de las mujeres de su entorno para el cuidado de sus hijos y tienen fuertes conflictos de pareja (37\%). La adherencia al tratamiento mostró dificultades que dirigen las conclusiones del estudio a la urgente necesidad de implementar programas constantes de educación a pacientes, cuidadores, familiares y los entornos inmediatos del niño con asma. Así como a favorecer la integración del varón en el cuidado de sus hijos y la comunicación con el equipo sanitario.
\end{abstract}

Palabras clave: Cuidadoras, asma descontrolada, pediátrico.

ABSTRACT. This study evaluated in caring mothers of children with poorly controlled asthma, parental perception of symptoms, family impact of asthma and socio-demographic aspects associated with trouble following medical prescriptions. Involving 110 primary caregivers (mean age $=37.6$ aged, $S D=6.7)$ and their children with asthma ( 7 and 15 ages, mean $=12.3$ years, $S D=2.9)$. The results showed that families are disadvantaged social class, and that the perception of symptoms occurs in high percentage (95.6\%). The caregivers showed a strong impact on their lives at the functional and emotional level (91\%). Regarding the socio-demographic are women with poor professional development, who have dropped out their formal working life. Fathers involved a little in the care of their children and caregivers received support from the women around, they frequently reported marital conflict (37\%). Treatment adherence showed difficulties who lead the study conclusions to the urgent need to implement patient education constants programs, caregivers, family and the immediate surroundings of the child with asthma, and to encourage the integration of men in care of their children and communication with the healthcare team.

Key word: Caregivers, uncontrolled asthma, pediatrics.

\section{INTRODUCCIÓN}

Diversas investigaciones en el ámbito de la salud confirman que la familia es la principal proveedora de cuidados para las personas enfermas, y en ocasiones constituye la única fuente de los mismos. En la literatura actual se pueden encontrar dos aspectos relacionados con la falta de equidad en el cuidado de los enfermos crónicos: el género y la clase social. Esto se manifiesta al observar que el cuidado de los pacientes es denominado socialmente como «asunto de mujeres»; quienes en su mayoría han sido caracterizadas como personas pobres y con empleos de salarios bajos. Por esta razón a partir de ahora, al referirnos a la muestra participante en este estudio se les denominará como «las cuidadoras»; pues en México, el cuidado de los enfermos sigue siendo una tarea femenina y más aún cuando el enfermo es un niño.

El cuidado de un enfermo en la familia o en casa supone un fuerte impacto en la vida de las cuidadoras que implica grandes costos. Recientes estudios han detectado que ellas se ven limitadas en el acceso a empleo, pues es común que la esfera de trabajo se vea afectada por bajas laborales constantes, abandono temporal o definitivo de la actividad remunerada, según la necesidad del enfermo a su cuidado. Estas 
restricciones hacen que las cuidadoras tengan un pobre desarrollo profesional, relaciones sociales limitadas, poca disponibilidad de tiempo propio, mala salud y repercusiones económicas, viendo deteriorada severamente la funcionalidad familiar $y$ su calidad de vida., ${ }^{1,2}$

En general, las cuidadoras y familiares de pacientes con enfermedades crónicas requieren capacitación y una atención integral por parte del equipo de salud para poder llevar a cabo su labor. Más aún, en enfermedades como el asma infantil, que tienen una alta incidencia y una prevalencia creciente. Las cuidadoras requieren ser atendidas con urgencia, ${ }^{3}$ tanto por los altos costos que genera la enfermedad como por el sufrimiento ocasionado, el cual se ha visto reflejado en los bajos índices de calidad de vida, ya sea de los pacientes como de ellas. ${ }^{4,5}$

La investigación actual sobre asma estudia temas como la relación entre el impacto de la enfermedad con la deficiente percepción parental de síntomas, la inadecuada medicación y el mal uso de servicios sanitarios, y ha explorado también su relación con variables como el género, la clase social y la edad; pero los resultados son aún inconsistentes, por lo que estas variables deben seguir siendo exploradas. ${ }^{2,6-9}$

Otras investigaciones latinoamericanas como las realizadas en Cuba y Chile, ${ }^{10,11}$ muestran que un elevado porcentaje de niños perciben deterioro en su vida cotidiana y que las dimensiones de calidad de vida afectadas por la enfermedad difieren en ambos países. Este hecho, en ambos estudios, puede ser atribuido a diferencias culturales, motivo suficiente para realizar estudios que consideren variables sociodemográficas y personales para conocer más acerca de las cuidadoras y que puede estar relacionado con el grado de seguimiento de las prescripciones médicas.

En estudios recientes sobre el impacto familiar del asma bronquial en México realizados por nuestro equi- po de investigación, llamó la atención que al ir captando pacientes, la mayoría de las cuidadoras de niños que llegaban en estado grave afirmaban no obtener suficientes beneficios del tratamiento farmacológico, así que los niños fueron valorados por el neumólogo quien identificó un elevado número de pacientes con «asma descontrolada» (ver participantes en método).

La Iniciativa Global para el Asma (GINA, del inglés Global Initiative for Asthma) ${ }^{12}$ proporciona dos sistemas de clasificación para esta enfermedad; el primero se centra en la severidad (intermitente, media persistente, moderada persistente) de la enfermedad (intermitente, persistente leve, persistente moderada, persistente severa); mientras que el segundo (el más reciente), se basa en el nivel de control del asma (controlada, parcialmente controlada o fuera de control) (tabla 1).

La iniciativa GINA ha desarrollado una serie de recomendaciones con la finalidad de mejorar la calidad de la atención de los pacientes con asma, y reducir sus problemas en varios ámbitos que se convierten en un fuerte dispendio económico para los sistemas de salud pública. Sin embargo, a pesar de todos los avances médicos y clínicos, aún es frecuente encontrar pacientes con grandes problemas para permanecer asintomáticos.

En México, la investigación sobre la calidad de vida o impacto familiar del asma bronquial, en general, es todavía muy reciente, situación que hace que existan pocos estudios que proporcionen información sobre las dificultades que viven las cuidadoras de niños con asma. Este hecho dio pauta para realizar una entrevista con las cuidadoras de este grupo de niños con asma descontrolada, pues al respecto hay menos información.

Según GINA ${ }^{12}$ y el Instituto Estadounidense del Corazón, Pulmón y Sangre (del inglés National Heart, Lung, and Blood Institute-NHLBI), ${ }^{13,14}$ los niveles de control

Tabla 1. Criterios utilizados para definir el asma no controlada.

\begin{tabular}{ll}
\hline \multicolumn{1}{c}{ Características } & Asma descontrolada \\
\hline Síntomas diurnos & Diario \\
Síntomas nocturnos o al despertar & Más de una vez a la semana \\
Limitación de actividades & Sí \\
Necesidad de medicamento de rescate & Sí \\
Función pulmonar $\left(\mathrm{FEV}_{1}\right)$ & $<80 \%$ \\
Exacerbaciones & Más de tres al año \\
\hline
\end{tabular}

Abreviaturas: $\mathrm{FEV}_{1}=$ Volumen espiratorio forzado en el primer segundo

Fuente: GINA $2011^{12}$

Los pacientes fueron considerados en la muestra cuando cumplían con tres o más características y cuando informaban más de tres exacerbaciones al año. 
del asma pueden oscilar entre asma controlada, parcialmente controlada o descontrolada según los síntomas diarios, limitaciones de actividades físicas, síntomas nocturnos, exacerbaciones, necesidad de medicación de rescate y función pulmonar.

En el presente estudio se observó que de la muestra total captada, $19.5 \%$ tenía asma descontrolada, lo que motivó a plantear los siguientes objetivos de investigación con el fin de identificar la problemática que enfrentan las cuidadoras de este tipo de pacientes en el intento de controlar el padecimiento: Primero, se identificó el perfil sociodemográfico de las 110 madres cuidadoras de niños con asma y sus familias, para lo cual se examinaron las variables de tipo personal (edad de la cuidadora, grado académico y estado civil), familiar (tipo de familia-nuclear/ extensa, número de hijos), laboral (ocupación, horas de trabajo, días de descanso a la semana, flexibilidad en el trabajo). Segundo, se evaluó la percepción de síntomas y el impacto familiar del asma para terminar identificando los principales problemas que estas cuidadoras tienen para adherirse al tratamiento de sus hijos con asma.

\section{MATERIAL Y MÉTODOS}

\section{Participantes}

La presente investigación consideró una submuestra $(19.5 \%)$ de un total de 564 pacientes captados. Participaron 110 cuidadoras (con edades entre 26 y 48 años; media de 37.60 años) y sus hijos con asma (68.1\% niños y $31.9 \%$ niñas, con edades entre 7 y 15 años de edad media = 13.6; desviación típica [DT] $=2.3$ ). La clase social de las familias se distribuyó en los tres primeros niveles del índice Hollinshead, ${ }^{15}$ que se basa en dos indicadores: ocupación y grado académico.

Criterios de inclusión: Pacientes que fueron identificados por el neumólogo como pacientes con asma descontrolada con base en la clasificación de GINA. ${ }^{12}$ Además, se consideró el volumen espiratorio forzado en el primer segundo $\left(\mathrm{FEV}_{1}\right)$, dato de la espirometría basal, el cual se refiere a la fracción de volumen que se expulsa en el primer segundo del esfuerzo respiratorio máximo, y casi siempre representa aproximadamente $80 \%$ del volumen total de aire en la espiración forzada (FVC). Otro dato espirométrico es la relación $\mathrm{FEV}_{1} / \mathrm{FVC}$ donde una disminución por debajo de $80 \%$ del valor normal indica un proceso obstructivo; asimismo, la variabilidad del $\mathrm{FEV}_{1}$ en el mismo día refleja la gravedad del asma y puede ser un indicador del grado de control de la enfermedad.

\section{Ambiente}

Se llevó a cabo la investigación en el servicio médico de las clínicas vinculadas a la Universidad Autónoma del Estado de México (UAEM), como parte de un estudio más amplio.

\section{Instrumentos}

\section{El grado de afectación:}

La gravedad del asma fue evaluada por un neumólogo pediatra estableciendo tres grados de severidad asmática: asma episódica, asma persistente moderada y asma persistente severa de acuerdo a los criterios establecidos por GINA.12 El neumólogo consideró asma descontrolada cuando el paciente informó tres de las siguientes características: síntomas diurnos diarios, síntomas nocturnos o despertar más de una vez a la semana, limitación de actividades, necesidad de medicamento de rescate, función pulmonar $\left(\mathrm{FEV}_{1}\right)$ menor $80 \%$ y presencia de exacerbaciones (más de tres veces por semana).

\section{Variables sociodemográficas:}

Se evaluó la variable clase social mediante el índice Hollingshead ${ }^{15}$ basado en dos indicadores: ocupación y grado académico. Dicho índice ordena esta variable en una escala de cinco puntos, ubicando en el nivel I la clase más baja y en el nivel $\mathrm{V}$ la clase más alta. También se recogieron las variables edad y género del paciente. Variables personales de la cuidadora y variables sobre la estructura familiar y sobre su actividad laboral.

\section{Dificultad de las cuidadoras para controlar la enfermedad:}

Se empleó una entrevista clínica donde se exploró: la administración del tratamiento farmacológico, la identificación, reducción/eliminación de factores de riesgo, la percepción de apoyo familiar, sus redes sociales de apoyo en el control de la enfermedad y la calidad de su comunicación con el equipo médico.

\section{Percepción parental de síntomas e incapacidad infantiles:}

Se evaluó mediante el «Cuestionario Percepción Parental de Síntomas e Incapacidad en Asma» (PSI), ${ }^{6}$ este instrumento unidimensional es la validación en población mexicana de la versión desarrollada en España, 
se dirige al cuidador primario, contiene 14 ítems, sus categorías de respuesta están codificadas en una escala Likert con las opciones que van de cero («nunca») a cuatro («todos los días»).

\section{Impacto familiar del asma:}

El «Cuestionario Impacto Familiar del Asma Bronquial Infantil-Revisado» (IFABI-R) ${ }^{4}$ se empleó para evaluar el impacto familiar del asma en las cuidadoras (en los últimos tres meses). Este cuestionario es la versión validada en México, va dirigido a la cuidadora primaria y consta de 18 ítems distribuidos en 4 dominios: funcional (ítems 1 a 3), estilo educativo (4 y 17), socioemocional (ítems 5 a 13, 16 y 18) y laboral (14 y 15). Sus categorías de respuesta están codificadas en una escala Likert con opciones que van de 1 (que significa menor impacto familiar) a 4 (que significa mayor impacto familiar).

\section{Procedimiento}

La investigación fue aprobada por el Comité Ético de la UAEM para trabajar en los hospitales vinculados a ella. Los participantes fueron seleccionados de una muestra total de 564 familias. Se les invitó a participar en esta investigación al haber identificado que su paciente tiene asma descontrolada y firmaron el consentimiento informado. El personal sanitario evaluó las variables clínicas $\mathrm{FEV}_{1}$ y grado de afectación. El neumólogo diagnosticó a los niños con asma descontrolada. Se entrevistó a las cuidadoras y se aplicaron los tres instrumentos: ficha de datos sociodemográficos, PSI e IFABI-R.

\section{Análisis de resultados}

Se realizó el estudio descriptivo de las características sociodemográficas iniciando con la clase social de las familias, se estudiaron las variables personales de la cuidadora (edad, grado académico y estado civil), la estructura familiar (tipo de familia-nuclear/extensa, número de hijos, clase social) y las variables laborales (ocupación, horas de trabajo, días de descanso a la semana y flexibilidad en el trabajo). También se describieron los resultados del PSI, del IFABI-R y se analizaron las dificultades de las cuidadoras para controlar el asma de sus hijos; para ello se consideró la administración del tratamiento farmacológico, la identificación reducción y/o eliminación de factores de riesgo, percepción de apoyo familiar/redes sociales de apoyo en el control de la enfermedad y la comunicación con el equipo médico.

\section{RESULTADOS}

En un inicio se describen las variables personales y familiares de las cuidadoras. Las variables personales mostraron que la edad de las madres de esta muestra osciló entre 26 y 48 años (media $=37.60$; DT $=6.78$ ). En cuanto al grado académico, sólo el $6.2 \%$ de las madres ha logrado concluir una carrera universitaria; $37.2 \%$ tiene bachillerato o secundaria truncos; $33.6 \%$ apenas tiene primaria trunca, y el restante $23 \%$ no tiene estudios. La variable estado civil se distribuyó en $89.4 \%$ de cuidadoras casadas (de las cuales $37 \%$ dijo vivir actualmente con graves conflictos de pareja a causa del asma de sus hijos). Diez punto seis por ciento son solteras, separadas o divorciadas. Sobre el tipo de familia y la clase social, $82.3 \%$ se agrupa en familias de tipo nuclear y $17.7 \%$ en familias de tipo extenso. El número de hijos se distribuyó de la siguiente manera: $7 \%$, 1 hijo; $71.7 \%$, 2 hijos; $21.2 \%$, más de 3 hijos. La clase social se distribuyó en los primeros tres puntos de la escala del índice Hollinshead, ${ }^{15}$ arrojando que la población pertenece a clases desfavorecidas socialmente.

Respecto a las variables laborales, se observó que en cuanto a la ocupación: $34 \%$ de las cuidadoras informaron no trabajar; sin embargo, cuando se exploró el impacto familiar del asma en el área laboral (ítems 14 y 15) de ese porcentaje, la mitad acababa de dejar su empleo para quedarse en casa a cuidar a su hijo enfermo; la otra mitad había cambiado su actividad laboral, pasando de ser trabajadoras asalariadas a ser trabajadoras del comercio informal. Aunque ni ellas ni sus familiares consideraban esta actividad como «un trabajo». Cincuenta y nueve por ciento tiene un trabajo operativo o subespecializado y sólo $7 \%$ algún cargo especializado o un negocio pequeño propio. Las cuidadoras informaron que las horas semanales de trabajo remunerado se distribuyen de la siguiente manera: 34\% que había afirmado «no trabajar» informó que dedicaba de 20 a 30 horas a la semana a su actividad de comercio. Cincuenta y siete por ciento informó trabajar 40 horas, el restante $9 \%$ trabaja entre 40 y 46 horas a la semana. En cuanto a los días de descanso a la semana: $34 \%$ de mujeres dedicadas al comercio dijeron que no cuentan con días de descanso. Seis por ciento dijeron tener 1 día de descanso y el restante $60 \%$ dijeron descansar dos días a la semana. Sobre la flexibilidad en el trabajo, $60 \%$ dijeron no tener flexibilidad en él y expresaron ser hostilizadas por compañeros y jefes por pedir permisos constantemente para llevar a sus hijos al médico.

En lo que respecta a la Percepción parental de síntomas e incapacidad, el PSI mostró que la muestra tiene una alta percepción de síntomas, ya que el porcentaje de cuidadoras que han percibido algún síntoma o algún indicador de incapacidad es de $95.6 \%$. 
En la variable Impacto familiar, el IFABI-R mostró que las madres perciben un alto impacto del asma en su calidad de vida (media $=3.28$ ). El dominio más afectado es el funcional, después el socioemocional y el estilo educativo; en menor grado se observa afectado el dominio laboral. Las cuidadoras explicaron que su desempeño laboral «ya no se ve afectado», algunas lo afirman, porque han dejado de trabajar y otras porque han modificado su actividad formal remunerada a una actividad de comercio informal en sus casas.

En cuanto a la variable Adherencia al tratamiento, cuando las madres tienen problemas para adherirse al tratamiento farmacológico (tabla 2), uno de los temas que más aluden es la complejidad del tratamiento y la falta de comprensión sobre el asma por parte de la familia, lo que hace que sus familiares subestimen los síntomas y sean poco colaboradores en la adherencia al tratamiento. En cuanto a la percepción de síntomas, se observó que las madres tienen dificultad para percibir el silbido de pecho o la opresión torácica como inicio de una exacerbación de asma, y acuden (para no equivocarse) siempre al servicio de urgencias, reconociendo que en ocasiones no era necesario. También se contempló que las cuidadoras no saben con certeza la función de los medicamentos. Informaron que asisten con frecuencia a la medicina alternativa o remedios caseros, interrumpiendo el tratamiento alópata.

En cuanto a los factores de riesgo se apreció que las cuidadoras no tienen suficiente conocimiento sobre la identificación de factores ambientales desencadenantes, aspecto que está estrechamente vinculado con la deficiente percepción de síntomas y con el mal uso de los servicios sanitarios. De la misma manera, el hecho de que las madres no comprendan la función y el efecto esperado de cada medicamento puede provocar que ellas no se adhieran al tratamiento (tabla 2).

Respecto al ámbito social de las cuidadoras (tabla 2), éstas reciben más apoyo de las mujeres de su familia que de sus propias parejas. La economía familiar se ve disminuida por los fuertes gastos que genera la medicación del niño enfermo. Asimismo, las madres informaron que les es difícil convencer a la familia para modificar hábitos y estilos de vida que favorezcan los cuidados especiales que el niño requiere para controlar los factores de riesgo (tabla 3 ).

En cuanto al apoyo del equipo médico, perciben que los médicos son sensibles a su problemática; sin embargo, no les dan una solución clara a los problemas que tienen para medicar a sus hijos, por ejemplo, cómo administrar aerosoles a niños pequeños, o cuando expresan que los esquemas de tratamiento son «muy complicados». En cuanto a la relación interpersonal, reconocen en la figura de la enfermera un apoyo im- portante para lograr los objetivos de tratamiento, pues son quienes les auxilian en la administración de inhaloterapia ambulatoria y otras terapias (tabla 3).

\section{DISCUSIÓN}

A partir de los datos recogidos con las cuidadoras de niños con asma, se puede observar que en México el cuidado de los hijos enfermos sigue siendo un «asunto femenino", lo que implica una baja participación de los varones de la familia, conflictos de pareja y un fuerte deterioro en la calidad de vida de las cuidadoras como lo habían identificado ya algunas investigaciones. ${ }^{1,16-18}$ Este hecho se corrobora al observar el bajo nivel educativo y las bajas o nulas expectativas de tener un trabajo formal o de volver a estudiar. En estas mujeres, el cuidado de un hijo enfermo ocasiona que ellas abandonen su desarrollo personal (laboral y/o profesional), siendo obligadas por las circunstancias a buscar actividades remuneradas en el "comercio informal». Mediante las entrevistas se observó que las madres cuidadoras subvaloran la actividad laboral que realizan - por tratarse de comercio informal-, pues ni ellas ni sus familias lo consideran «trabajo», generando en algunas de ellas tensiones y resentimientos respecto a sus parejas. Esta situación ha estado presente en más de una de nuestras investigaciones. ${ }^{4}$

Según las cuidadoras, el asma de sus hijos también se relaciona con un deterioro en sus relaciones de pareja, pues casi la mitad de las que están casadas expresaron que viven graves conflictos con sus esposos a causa del asma de sus hijos; tal como lo aseveran Rydström et al., en un estudio donde se observa cómo las familias son sometidas a un estado constante de incertidumbre que afecta sus relaciones. ${ }^{17}$ Las madres que viven solas refieren como uno de los motivos más importantes de la separación, la enfermedad de sus hijos. Como se puede observar, es imponderable la necesidad de desarrollar estrategias para incorporar al padre en el cuidado del hijo enfermo y en general de sus hijos. Si bien es cierto que los padres son comúnmente el proveedor económico mayoritario de la familia y que los gastos por la enfermedad son muchos, se debe insistir en el desarrollo de políticas sociales que apoyen su participación; por ejemplo, en México son pocas las instancias públicas que proporcionan licencias de «cuidados maternos», y en los pocos casos que se dan, como su nombre lo dice, dejan excluidos a los padres de gozar de este beneficio.

Sumado a lo anterior, se observó que las cuidadoras y sus familias justifican la baja implicación del padre en el cuidado del hijo enfermo con el factor económico - pues ellos son proveedores-. Sin embargo, conside- 
Tabla 2. Problemas de las cuidadoras en la adherencia al tratamiento e impacto familiar del asma descontrolada.

\begin{tabular}{|c|c|c|c|}
\hline $\begin{array}{l}\text { Administración del tratamiento } \\
\text { farmacológico }\end{array}$ & $\begin{array}{l}\text { Identificación, disminución/eliminación } \\
\text { de factores de riesgo }\end{array}$ & $\begin{array}{l}\text { Percepción de } \\
\text { síntomas/mejoría }\end{array}$ & $\begin{array}{l}\text { Impacto familiar del } \\
\text { asma }\end{array}$ \\
\hline 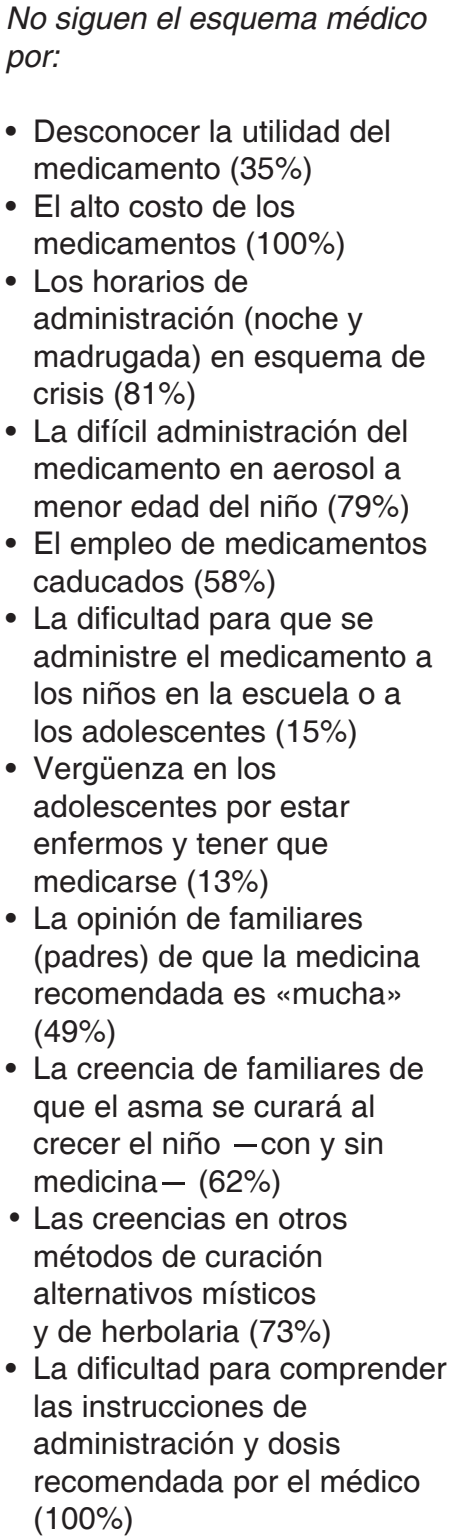 & $\begin{array}{l}\text { Ambientales: } \\
\text { No identifican qué es lo que } \\
\text { desencadena una crisis (100\%) } \\
\text { Alergias: } \\
\text { Confusión al identificar los alimentos } \\
\text { alergénicos (16\%) } \\
\text { Higiene en el hogar: } \\
\text { Confusión al eliminar o cambiar líquidos } \\
\text { limpiadores y aromatizantes en casa } \\
\text { (79\%) } \\
\text { Sin presupuesto para cambiar sitios } \\
\text { decorados con tapetes y cortinas ( } 77 \%) \\
\text { No usan aspiradoras teniéndola (17\%) } \\
\text { No tienen aspiradoras ( } 27 \% \text { ) } \\
\text { Hábitos nocivos: } \\
\text { Padres fumadores y niños expuestos } \\
\text { al humo del tabaco (34\%) } \\
\text { Contacto estrecho con mascotas ( } 49 \%) \\
\text { Restricción de actividades: } \\
\text { Evitan el ejercicio físico en los niños } \\
\text { para evitar crisis (18\%) } \\
\text { jugitan que los niños salgan a la calle a } \\
\text { frío ( } 73 \% \text { ) }\end{array}$ & $\begin{array}{l}\text { Las cuidadoras no } \\
\text { saben si hay «silbi- } \\
\text { do» de pecho o no, } \\
\text { acuden siempre a } \\
\text { Urgencias (61\%) } \\
\text { La medicación no } \\
\text { siempre funciona, le } \\
\text { doy el «gas» (algún } \\
\text { broncodilatador) y } \\
\text { mejora un ratito, pero } \\
\text { la mejoría no dura } \\
\text { (13\%) } \\
\text { Le dan jarabe que } \\
\text { «sólo hace más } \\
\text { flemas» (ambroxol) } \\
\text { (19\%) } \\
\text { Cuando «no mejoran } \\
\text { pronto» llevan a los } \\
\text { niños a tratamientos } \\
\text { alternativos (inhala- } \\
\text { ciones de vapores de } \\
\text { hierbas) (39\%) }\end{array}$ & $\begin{array}{l}\text { El área más } \\
\text { afectada es el área } \\
\text { socioemocional } \\
(97 \%) \\
\text { En segundo término } \\
\text { es el área funcional } \\
(91 \%) \\
\text { Las áreas: laboral } \\
\text { y estilo educativo } \\
\text { recibieron un menor } \\
\text { impacto (38 y } 45 \% \text {, } \\
\text { respectivamente) }\end{array}$ \\
\hline
\end{tabular}

Porcentajes de la problemática en el seguimiento del tratamiento integral que enfrentan las madres cuidadoras de niños con asma descontrolada.

ran, por un lado, que los padres no apoyan el tratamiento y muchas veces se oponen a él debido a que nunca les acompañan a las consultas médicas ni conocen los esquemas de administración de los fármacos y tampoco participan en la prevención de riesgos en el hogar; por ejemplo, muchos de ellos fuman y no ven necesario modificar este hábito. Por otro lado, las cuidadoras informan sentirse abandonadas por su pareja y sólo encuentran apoyo en las mujeres de su familia y círculos sociales femeninos más cercanos. Otra problemática importante que las madres detectan es la necesidad de educar a los entornos inmediatos del niño con asma (incluir escuelas, comunidad y centros de actividades extraescolares) para fomentar el trato adecuado de los niños, quienes en la mayoría de los casos son limitados en sus actividades escolares y recreativas por su condición de salud. 
Tabla 3. Aspectos sociales de las cuidadoras de niños con asma descontrolada
Apoyo familiar y social
Comunicación con el equipo médico

\section{Aspectos positivos:}

- Abuelas, tías, amigas y vecinas apoyan a la madre en el cuidado del niño cuando está enfermo (87\%)

- El padre casi nunca se queda a cuidar al niño enfermo $(98 \%)$

- La familia es solidaria con el gasto, se disminuyen gastos para tener recursos para la medicina (73\%)

Aspectos negativos:

- La familia niega o minimiza el diagnóstico (29\%)

- La familia se niega a hacer cambios en su estilo de vida; p. ej., padres que siguen fumando (57\%)

- Cuando el niño presenta crisis nadie se queda con la cuidadora para atender al niño enfermo. Sólo recibe compañía de la familia (mujeres solamente) si el niño es hospitalizado $(69 \%)$
Aspectos positivos:

- Los médicos se muestran dispuestos para explicar los aspectos clínicos de la enfermedad y la administración de medicamentos (95\%)

- El personal de enfermería es claro y amable asiste a las madres para poder proporcionar a sus hijos terapia respiratoria $(99 \%)$

Aspectos negativos:

- Los procedimientos de medicación son complejos, afirman el $75 \%$ de las cuidadoras, pues se tienen que dar varios medicamentos en diferentes horarios y la terapia vía inhalada presenta dificultades (p. ej., la administración de dichos medicamentos en niños pequeños)

- Los médicos no ofrecen alternativas de solución para facilitar los procedimientos de administración de medicamentos vía inhalada, tanto en niños muy pequeños como en adolescentes (35\%)

Porcentajes de los aspectos positivos y negativos que se perciben el ámbito familiar, social, con el personal de salud, las cuidadoras de niños con asma descontrolada.

En relación a la comunicación con el equipo sanitario, ésta debe mejorarse reorientando la información y estrategias de educación para la salud empleada por los médicos, quienes podrían incluir en el equipo médico multidisciplinario al psicólogo de la salud. Profesional que entre sus funciones tiene la educación de los pacientes mediante estrategias especializadas que coadyuvan a una modificación de estilos de vida, mayor apego al tratamiento farmacológico y al control ambiental para prevenir factores de riesgo.

Como se puede observar a lo largo de este estudio, son muchos los factores en la vida cotidiana de las mujeres cuidadoras que pueden estar influyendo estrechamente en el cuidado de sus hijos con asma. A pesar de que las más actuales guías internacionales del manejo de esta enfermedad intentan incluir dichos aspectos, se sabe aún muy poco sobre ellos. ${ }^{2,3,18}$

\section{CONCLUSIONES}

Para las cuidadoras mexicanas de niños con asma, este estudio constituye un primer paso a reconocer la complejidad de su vida cotidiana, que dará pauta para estructurar intervenciones mejor orientadas ${ }^{6}$ y más adelante, quizá, a la generación de políticas sociales favorecedoras para la inclusión del varón en el cuidado de los hijos enfermos.
A este respecto se debe señalar que en Latinoamérica hay mucho que hacer sobre la incorporación de los varones en el cuidado de los hijos (tanto sanos como enfermos), acción que puede favorecer la calidad de vida de las cuidadoras y evitar los problemas de pareja y familiares detectados en la población del estudio.

En este sentido, las intervenciones en nuestro país deben dirigirse a favorecer dos aspectos centrales: en primer lugar, educar el entorno inmediato de los niños para evitar los factores de riesgo, lo cual lleva a considerar estrategias educativas no sólo para la familia, sino para la escuela y la comunidad. En segundo lugar, orientar esfuerzos a la creación de políticas sociales para favorecer el ejercicio de una paternidad más plena, como la creación de licencias de «paternidad» para apoyar cuando nacen los hijos o cuando éstos están enfermos, las cuales existen ya en países europeos con excelentes resultados.

En consecuencia, dada la importancia del entorno sociocultural en estos temas, se sugiere desarrollar investigaciones de este tipo en otros países para poder estudiar más a fondo las particularidades de la problemática de las cuidadoras de niños con asma en diferentes entornos culturales y sociales, ${ }^{16}$ lo que permitirá intervenciones más exitosas que eleven la calidad de vida de las cuidadoras y sus familias. 


\section{REFERENCIAS}

1. García-Calvente MM, Mateo-Rodríguez I, Eguiguren AP. El sistema informal de cuidados en clave de desigualdad. Gac Sanit 2004;18(Supl 1):132-139. Fecha de consulta: agosto, 2013. Disponible en: http:// scielo.isciii.es/scielo.php?script=sci_pdf\&pid=S0213$91112004000400021 \&$ lng $=$ es\&nrm=iso\&tlng=es

2. Brazil K, Krueguer P. Patterns of family adaptation to childhood asthma. J Pediatr Nurs 2002;17(3):167-173.

3. Calam R, Gregg L, Simpson B, Morris J, Woodcock A, Custovic A. Childhood asthma, behavior problems, and family functioning. J Allergy Clin Immunol 2003;112(3):499-504.

4. Bazán-Riverón GE, Torres-Velázquez LE, Prat-Santaolaria R, Sandoval-Navarrete J, Forns-Serrallonga D. Impacto familiar del asma pediátrica. Versión mexicana del cuestionario IFABI-R. Rev Inst Nal Enf Res Méx 2009;22(2):73-83.

5. Bazán-Riverón GE, Prat-Santaolaria R, Torres-Velázquez LE, Sandoval-Navarrete J, Forns-Serrallonga D. Asma pediátrica: calidad de vida del paciente relacionada con el impacto familiar. Neumol Cir Torax 2010;69(2):75-83.

6. Bazán-Riverón GE, Forns-Serrallonga D, Prat-Santaolaria $\mathrm{R}$, et al. Validación del cuestionario "Percepción parental de síntomas infantiles de asma en México". Rev Inst Nal Enf Res Méx 2008;21(3):169-180.

7. Chen E, Hanson MD, Paterson LQ, Griffin MJ, Walker HA, Miller GE. Socioeconomic status and inflammatory processes in childhood asthma: the role of psychological stress. J Allergy Clin Immunol 2006;117(5):1014-1020.

8. Guyatt GH, Juniper EF, Griffith LE, Feeny DH, Ferrie PJ. Children and adult perceptions of childhood asthma. Pediatrics 1997;99(2):165-168.

9. Juniper EF, Guyatt GH, Feeny DH, Ferrie PJ, Griffith LE, Townsend M. Measuring quality of life in the parents of children with asthma (PACQLQ). Qual Life Res 1996;5(1):27-34.

10. Gómez BRA, González YY, Morejón FJM, Travieso PR. Calidad de vida relacionada con salud en niños asmáticos y sus cuidadores. MediSur (en línea) 2005;3(3):13-26. Fecha de consulta: octubre, 2013. Disponible en: http:// redalyc.org/articulo.oa?id $=180019788003$ ISSN

11. Vidal GA, Duffau TG, Ubilla PC. Calidad de vida en el niño asmático y su cuidador. Rev Chil Enferm Respir 2007;23(3):160-166.
12. Global Initiative for Asthma. Global strategy for asthma management and prevention. Revised 2011, Md., National Institute of Health, National Heart, Lung and Blood Institute. Publication. Fecha de consulta: junio, 2013. Disponible en: http://www.ginasthma.org/uploads/users/files/GINA_Report2011_May4.pdf.

13. National Institutes of health, National Heart, Lung and Blood (NHLBI). Guidelines for the diagnosis and management of asthma. 2007. Fecha de consulta: Septiembre 2011. Disponible en: http://www.nhlbi.nih.gov/guidelines/asthma/asthgdln.htm

14. Consenso Latinoamericano sobre el Asma de Difícil Control. Actualización. Drugs of Today 2008, Vol. XX, Supl. X. Fecha de consulta: Julio, 2011. Disponible en: http://www.cmdlt.edu.ve/pdfs_ciencias/consLatinAsma. pdf.

15. Hollingshead AB. Four factor index of social status: Unpublished manuscript. USA: Yale University, Department of Sociology; 1979, p.387-397.

16. Bazán RG. Evaluación del impacto familiar del asma bronquial infantil (tesis). España: Universidad Autónoma de Barcelona, España; 2009. Disponible en: www.tdx. cat/bitstream/10803/5496/1/gebr1de1.pdf

17. Rydström I, Dalheim-Englund AC, Segesten K, Rasmussen BH. Relations governed by uncertainty: part of life of families of a child with asthma. J Pediatr Nurs 2004;19(2):85-94.

18. Gustafsson D, Olofsson N, Andersson F, Lindberg B, Schollin J. Effect of asthma in childhood on psychosocial problems in the family. J Psychosom Res 2002;53(6):1071-1075.

\section{$\triangle$ Correspondencia:}

Dra. Georgina Eugenia Bazán Riverón Grupo de Investigación: Enfermedades Crónicas. Unidad de Investigación Interdisciplinaria en Ciencias de la Salud y la Educación (UIICSE). División de Investigación y Postgrado. Facultad de Estudios Superiores Iztacala, UNAM.

Teléfono: (55)56231333, extensión 39806

Correo electrónico: gebrmx@yahoo.com.mx

Los autores declaran no tener conflictos de interés. 